\title{
Bayhealth, COVID-19 and Technology - Safely Discovering our New Normal
}

\author{
Richard Mohnk, MS \\ Chief Information Officer, Bayhealth Medical Center
}

As a regional health care leader, safety and high reliability are key elements of service excellence at Bayhealth. As we all continue to discover our new normal, COVID-19 is pushing health care into this new normal as well.

The first quarter of 2020 felt like a discovery of unknowns. Unknowns in how we treat COVID19 , how we manage patient care, where we place patients as we run out of beds, what will be allowed as it relates to visitors, how will we manage this crisis from our 24-hour command center, and how will we successfully work with state and federal guidance. Space, testing, supplies and understanding how to treat this new disease dominated those early days.

Technology was critical. Technology supported the opening of new care spaces at the Bayhealth Kent and Sussex campuses, as well as temporary locations near our emergency departments. These new spaces were immediately equipped with all the necessary telecommunications, computers, mobile devices, and Wi-Fi capabilities. While we were fortunate to not need many of the additional care spaces created, we were prepared. The need for technology extended beyond direct patient care. Testing and supplies required dashboards and reporting mechanisms to easily send information to the Bayhealth team and state health personnel so our staff could be equipped with the necessary safety supplies to continue caring for our community.

As the second and third quarters of 2020 rolled in telehealth, video conferencing, flex scheduling (working from home), safe distancing, patient monitoring, and visitation, staffing and security (ransomware/dual factor authentication/electronic prescriptions) became serious topics to address. Spring, summer and fall created significant opportunities for change. Patients needing telehealth suddenly required video visits, staff needing to collaborate required video conferencing - all as we navigated staff working remotely, changes to how clinicians prescribed medications, new methods for visitors to comfort family members and the future of a new Graduate Medical Education program on the horizon.

Video capabilities infiltrated most all aspects of health care. In person meetings were replaced with video conferences. Physician practices focused predominantly on telehealth visits. Cyber security was on everyone's mind with the risk of ransomware infiltration into vaccination efforts all around us. Dual factor authentication became the norm and helped security access and a reduction in opioid prescriptions. Hiring of new staff members suddenly became a video interview process. The reality is, technology made all this immediately possible. Because of our focus on technology at Bayhealth, we were able to seamlessly pivot when needed to adapt to a virtual world.

As time rolled into 2021 and the hope of vaccines became the rescue amid escalating COVID-19 cases, it was clear our efforts to introduce video visits with patients and monitoring with cameras for our care givers were going to be here to stay. Nursing could monitor patients safely with new camera technology and our patient advocates were making a huge difference using tablets to conduct video calls for patients and their families. These were even more examples of lasting impact leveraging technology will have on our community.

Article DOI: 10.32481/djph.2021.07.007, Copyright (c) 2021 Delaware Academy of Medicine / Delaware Public Health Association 
As we enter the summer of 2021, the Bayhealth technology team is proud to continue supporting our health system and our community every day.

Copyright (c) 2021 Delaware Academy of Medicine / Delaware Public Health Association.

This is an Open Access article distributed under the terms of the Creative Commons Attribution Non-Commercial License (https://creativecommons.org/licenses/by-nc-nd/4.0/) which permits unrestricted non-commercial use, distribution, and reproduction in any medium, provided the original work is properly cited.

Article DOI: 10.32481/djph.2021.07.007, Copyright (c) 2021 Delaware Academy of Medicine / Delaware Public Health Association 